\title{
Discriminating between the nearfield and the farfield of acoustic transducers
}

\author{
Kenneth G. Foote ${ }^{a)}$ \\ Woods Hole Oceanographic Institution, Woods Hole, Massachusetts 02543
}

(Received 7 July 2014; revised 24 August 2014; accepted 28 August 2014)

\begin{abstract}
Measurements of the performance of acoustic transducers, as well as ordinary measurements made with the same, may require discriminating between the farfield, where the field is spherically divergent, and the complementary nearfield, where the field structure is more complicated. The problem is addressed for a planar circular piston projector, with uniform normal velocity distribution, mounted in an infinite planar rigid baffle. The inward-extrapolated farfield pressure amplitude $p_{f}$ is compared with the exact nearfield pressure amplitude $p_{n}$, modeled by the Rayleigh integral, through the error $20 \log \left|p_{f} / p_{n}\right|$. Three sets of computations are performed for a piston with wavenumber-radius product $k a=10$ : normalized pressure amplitudes and error versus range at angles corresponding to beam pattern losses of $0,10,20$, and $30 \mathrm{~dB}$; error versus angle at three ranges, $a^{2} / \lambda, \pi a^{2} / \lambda$, and $10 a^{2} / \lambda$, where $\lambda$ is the wavelength; and range versus angle for each of two inward-bounded errors, 1 and $0.3 \mathrm{~dB}$. By reciprocity, the results apply equally to the case of a baffled circular piston receiver with uniform sensitivity over the active surface. It is proposed that proximity criteria for measurements of fields associated with circular pistons be established by like modeling, and that a quality factor be assigned to measurements on the basis of computed errors. (C) 2014 Acoustical Society of America. [http://dx.doi.org/10.1121/1.4895701]
\end{abstract}

PACS number(s): 43.20.Ye, 43.58.Vb, 43.20.Rz, 43.30.Xm [ADP]

Pages: $1511-1517$

\section{INTRODUCTION}

The farfield of an acoustic transducer used as a projector, to transmit sound, is that spatial region where the directpath pressure amplitude varies inversely with distance from the transducer. By reciprocity, this also applies to an acoustic transducer used as a receiver of sound, in which the directpath pressure amplitude at the transducer varies inversely with the distance to the acoustic source. ${ }^{1}$ The nearfield is the spatial region that is complementary to the farfield. Its extent is often gauged as a multiple of a characteristic transducer dimension or a multiple of the square of that dimension divided by the acoustic wavelength. These measures reflect the dependence of the nearfield-farfield transition distance on both amplitude and phase.

This understanding is quite general, but there is evident disagreement, sometimes admitted dissatisfaction, with the numerical values of the multipliers, not to mention their possible dependence on angle relative to the acoustic axis of the transducer. While this matter might appear to be mainly of academic interest, it has practical importance in many applications. It is also amenable to analysis, illustrated here for an idealized planar circular piston transducer, with uniform normal velocity distribution, or sensitivity, over the active acoustic surface, mounted in an infinite planar rigid baffle.

\section{A. Nearfield-farfield transition distance in review}

The following comments, if not otherwise qualified, apply to statements made specifically about the baffled

\footnotetext{
a) Author to whom correspondence should be addressed. Electronic mail: kfoote@whoi.edu
}

circular piston transducer operating harmonically, expressed in terms of the piston radius $a$ and acoustic wavelength $\lambda$. General distances are denoted by the range $r$; along the transducer axis, by $z$.

In an early work, Stenzel ${ }^{2}$ defined the axial farfield by the dual conditions $z \gg a$ and $z \gg \pi a^{2} / \lambda$. Rschevkin ${ }^{3}$ quoted Stenzel's second condition. Skudrzyk ${ }^{4}$ acknowledged Stenzel's work, but on the basis of a Huygens zone construction, with difference in distances from an axial field point to the center of the piston and to the rim of the piston, gave the axial transition distance as $a^{2} / \lambda$, also expressing this more accurately as $a^{2} / \lambda-\lambda / 4$.

Williams ${ }^{5}$ gave a single measure for the axial transition distance, namely, $a^{2} / \lambda$, citing an analogy with radiation from a planar piston and that from a Fresnel aperture, but also noting "several approximations of uncertain validity." Beyer and Letcher ${ }^{6}$ referred to Fresnel and Fraunhofer diffraction, with "a dividing line" between nearfield and farfield at $z=a^{2} / \lambda$. Zemanek ${ }^{7}$ referred to the same diffraction phenomena, but based on a numerical investigation established "a firm boundary between the near- and farfield." If $a / \lambda<13.3$, measurements could be made at $r<10 a$; if $a / \lambda>13.3$, measurements would have to be made at $r>10 a$. Pierce ${ }^{8}$ also referred to Fresnel and Fraunhofer diffraction regions according to Fresnel-Kirchhoff diffraction theory from optics, ${ }^{9}$ describing the farfield by the dual conditions $r \gg a$ and $r \gg 2 \pi a^{2} / \lambda$. Beranek ${ }^{10}$ gave similar conditions $r \gg 2 a$ and $r \gg \pi a^{2} / \lambda$. Also referring to Fresnel diffraction, Stansfield ${ }^{11}$ derived a condition for the nearfield-farfield transition distance, $r=4 a^{2} / \lambda$, but allowing that this could be "unnecessarily conservative."

In several well-known textbooks published in multiple editions, measures for the nearfield-farfield transition 
distance have evolved. In their first edition, Kinsler and Frey ${ }^{12}$ recognized spherical divergence beyond $r=2 a^{2} / \lambda$. In the third edition, Kinsler et al. ${ }^{13}$ gave the transition distance as $a^{2} / \lambda$, but with the qualification "roughly." In the fourth edition, ${ }^{14}$ the same distance, $r=a^{2} / \lambda$, was cited, but without qualification. Urick ${ }^{15}$ referred indirectly to the transition distance in the first edition of his textbook through a planewave parameter required for measurements at $r<\pi a^{2} / \lambda$. This was repeated in the second edition, ${ }^{16}$ but in the third edition, ${ }^{17}$ calibration of a projector was stated as being "always made in the farfield beyond $\left[\pi a^{2} / \lambda\right]$." Clay and Medwin ${ }^{18}$ referred to a critical range for the transition distance, $r=\pi a^{2} / \lambda$, as being "arbitrarily decided" by the American National Standards Institute (ANSI). In their second edition, Medwin and Clay ${ }^{19}$ simply stated that the farfield begins at $r=\pi a^{2} / \lambda$.

More recently, Blackstock ${ }^{20}$ defined the farfield as $r>\pi a^{2} / \lambda$ and $r \gg a$, but also noted that the quantity $\pi a^{2} / \lambda$ "roughly marks the end of the nearfield... and the beginning of the farfield," and that the underlying model "is a gross oversimplification and must not be taken too literally." Comments on the off-axis field imply a greater complexity. Sherman and Butler ${ }^{21}$ also commented on the off-axis field, through the beam pattern, gauging the beginning of the farfield as $r \geq 2 a^{2} / \lambda$. They added, a "more accurate condition... is sometimes necessary," describing this as $r \geq 4 a^{2} / \lambda$.

A very different approach was taken by Bobber, ${ }^{1}$ who initially addressed the on-axis case by computing errors associated with customary small-argument approximations of the exact nearfield expression. These analytically treated errors also prescribed conditions to be applied to axial field measurements at finite distances. Bobber then quoted standard criteria for a circular piston with uniform velocity distribution, namely, $z \gg \pi a^{2} / \lambda$ and $z \geq a$, but gave his own proximity criteria, relating these to errors incurred by the mentioned approximations. For maximum errors of $0,0.35$, and $0.90 \mathrm{~dB}$ due to replacement of the sine of an angle by the angle, the respective criteria were that $\mathrm{z}$ should equal or exceed $2 \pi a^{2} / \lambda, \pi a^{2} / \lambda$, and $2 a^{2} / \lambda$. For a maximum on-axis error of $0.5 \mathrm{~dB}$, Bobber gave proximity criteria with respect to phase and amplitude thus: $z \geq 4 a^{2} / \lambda$ and $z \geq 2 a$, characterizing these as "conservative criteria." Offaxis errors were bounded with respect to the endfire orientation, confirming the phase-proximity criterion but increasing the amplitude-proximity criterion to $r \geq 20 a$. This second criterion was modified by considering the effect of tapering of the Fresnel zone areas from the central zone, supporting the less stringent condition, $r \geq 10 a$.

All of the foregoing criteria apply to the case of a point receiver when a projector is being used or, by reciprocity, a point source when a receiving transducer is being used, including assumption of a uniform normal velocity distribution, or sensitivity, on the active acoustic area.

Bobber ${ }^{1}$ recognized other practical effects too, such as those of finite-size receiving transducers when projectors, or transmitting transducers, are being measured, as well as nonuniform distributions of velocity, or sensitivity, on the acoustically active transducer surface. $\mathrm{Sabin}^{22}$ provided guidelines for on-axis measurements when both transducers are circular pistons oriented normally to the line connecting their centers. Bobber and Sabin ${ }^{23}$ and Sorokin ${ }^{24}$ considered the analogous case when both transducers are finite-length cylindrical transducers approximated as finite lines. The case of non-uniform velocity, or sensitivity, distribution on the acoustically active surface of the transducer being measured was first treated by Stenzel $^{2}$ and subsequently by Greenspan $^{25}$ and Aarts and Janssen. ${ }^{26}$

The nearfield-farfield transition distance is also treated in the standards literature. In IEC $60565,{ }^{27}$ the main separation distance between two transducers was defined for two specified errors. For a circular piston projector and point receiver, the minimum axial distance needed to limit the error to $0.3 \mathrm{~dB}$ was defined by the criteria $z>4 a^{2} / \lambda$ and $z>10 a$. For an error of $0.2 \mathrm{~dB}$, these separation distances were increased by $20 \%$. For measurement of the beam pattern, the respective separation distances were to be doubled. In ANSI/ ASA S1.20-2012, ${ }^{28}$ the criteria were stated as $r>\pi a^{2} / \lambda$ and $r>a$, without specification of an error, citing Ziomek. ${ }^{29}$

\section{B. Importance of the nearfield}

The transducer nearfield is important in many applications and uses of sound. In biophysical studies and medical uses of ultrasound, e.g., diagnostic imaging and therapeutic hyperthermia, subject tissues may lie within the nearfield. Even if such tissues are in the transducer farfield, other, intervening tissues will be exposed to sound in the nearfield. ${ }^{30}$ The magnitude and location of peak pressures will be of concern if causing unwanted radiation effects, e.g., cavitation, which also limits radiated transducer power. ${ }^{31}$ It is also important for understanding interactions with biological media.

In fisheries acoustics, the transducer nearfield is generally avoided, but the entire trend of the field is toward maximal exploitation of technology in the quantification of organisms of all types and environment too. The transducer nearfield thus represents an opportunity to increase the acoustic sampling volume, ${ }^{32}$ but with proper allowance made for the detailed structure of the nearfield. In the case of a parametric sonar used to measure herring (Clupea harengus) in situ in the Norwegian Sea, ${ }^{33}$ it was impossible to avoid the nearfield. Admittedly, the array was virtual, existing in space away from the transducer, as the formation of an exceptionally directional beam at very low frequencies depends on the cumulative effect of the nonlinearity inherent in the medium on the propagating and interacting primary, high-frequency waves. ${ }^{34}$ A scheme for quantification of parametric sonar echoes has been developed, ${ }^{35,36}$ drawing on a computational model for the difference-frequency nearfield of the parametric sonar, i.e., the nearfield. ${ }^{37,38}$

In some underwater geophysical investigations, measurements are inevitably made in the nearfield, for example, with high-frequency sidescan sonars operating at short ranges. The method of dynamic focusing is used to restore phase coherence to echoes by shading-adjusting amplitude and phase - of elements in the sidescan sonar array. The same is true of multibeam sonar.

Microphones and loudspeakers in air also have nearfields, and consideration of these is a factor in their performance measurement. A case in point is calibration of 
microphones with circular membranes by the reciprocity method, which requires free-field conditions and avoidance of the nearfield. ${ }^{10}$

Common to all of these applications is knowledge of the nearfield-farfield transition. Apropos of measurements, this knowledge is essential for data interpretation. The same knowledge is essential in measurements of transducer performance, which are typically made according to protocols, with conditions imposed on the measurement distance relative to the transition distance. ${ }^{1,27}$ In these cited cases, it is recommended that the performance measurements be made in the transducer farfield. In some other cases, such as those involving very large transducers and arrays, special facilities have been built and configured to effectively simulate farfield conditions while operating in the nearfield. ${ }^{39-41}$

\section{Rationale and organization of study}

The amount of attention that the nearfield has received in the literature witnesses to its importance. Many transducers, whether used as projectors or receivers of sound, in air or water, are circular, with an approximately constant normal velocity distribution, or sensitivity, and mounting in a baffle that is effectively large and stiff. In such cases, modeling the transducer as a planar circular piston with uniform normal velocity distribution and mounting in an infinite planar rigid baffle is reasonable.

The radiation field of a baffled circular piston transducer as described here is modeled by the Rayleigh integral. This field is evaluated in the nearfield and compared with the inward-extrapolated farfield. The error in the extrapolated farfield relative to the nearfield is quantified for a piston with wavenumber-radius product $k a=10$ with the aim of elucidating the nearfield-farfield transition. Criteria on the field position for measurement of and with such transducers are stated for this case, but with generalizations too.

The choice of the relative transducer size $k a=10$ is expedient but also representative of circular piston transducers in use. The value for $k a$ is sufficiently low that the numbers of sidelobes and nulls are small, avoiding obscuring the nearfield wave-interference phenomena being studied. At the same time, the value $k a=10$ is well within the range of circular piston transducers that are being used. Three examples are cited. (i) The Panametrics V391 0.5-MHz transducer has a diameter of $29 \mathrm{~mm}$, with operating frequency range $100-1000 \mathrm{kHz}$, hence with $\mathrm{ka}$ in the nominal range 6-60. (ii) A Piezo Composite Transducer Ltd. custom-made piston transducer is divided into a central circular area of diameter $100 \mathrm{~mm}$ and contiguous annulus of effective outer diameter $135 \mathrm{~mm}$. Given the operating frequency range $20-500 \mathrm{kHz}$, the respective $k a$-ranges are roughly $4-105$ and 6-140. (iii) An Ultran WS150-0.25 transducer has a diameter of $30 \mathrm{~mm}$. Its reported operating frequency bands are 200-300 and $150-350 \mathrm{kHz}$, with respective $k a$ ranges of $13-19$ and 9-22.

\section{RADIATION FIELD OF A BAFFLED CIRCULAR PISTON}

The subject is a planar circular piston transducer, with uniform normal velocity distribution on the acoustically active surface, mounted in an infinite planar rigid baffle. For convenience, this is imagined to be an active source, i.e., a projector of sound. As noted, by reciprocity the field structure applies equally to the case that the transducer is used as a receiver of sound emanating from a point source at the field point. ${ }^{1}$

A general expression for the pressure field associated with a planar acoustic source mounted in an infinite rigid baffle is available through the Rayleigh integral, ${ }^{8,42}$ described by Sherman and Butler" ${ }^{21}$ as "one of the most frequently used equations in acoustics." It is given here essentially in the form derived by Pierce $^{8}$ for pressure $p$ at field position $\boldsymbol{r}$ for a harmonic wave of angular frequency $\omega$,

$$
p(\boldsymbol{r})=\frac{-i \omega \rho_{0}}{2 \pi} \int \boldsymbol{v}\left(\boldsymbol{r}^{\prime}\right) \cdot \hat{n} R^{-1} \exp (i k R) d S,
$$

where $\rho_{0}$ is the mean mass density of the immersion medium, $\boldsymbol{v}\left(\boldsymbol{r}^{\prime}\right)$ is the velocity of the piston at position $\boldsymbol{r}^{\prime}$ on the acoustically sensitive surface $S, \hat{n}$ is the unit normal to $S$ at $\boldsymbol{r}^{\prime}$, with $\hat{n} \cdot \boldsymbol{r}>0, R=\left|\boldsymbol{r}-\boldsymbol{r}^{\prime}\right|$ is the distance from a point on the piston surface to the field point, $k=\omega / c_{0}$ is the wavenumber, and $c_{0}$ is the speed of sound in the medium, assumed constant. This is equivalent to other expressions of the Rayleigh integral for harmonic waves, e.g., Ref. 21, but with the useful reminder through the term $-i \omega v$ that the radiation of sound is due to acceleration of the piston surface, ${ }^{20}$ not velocity per se.

For a uniform normal velocity $\boldsymbol{v}\left(\boldsymbol{r}^{\prime}\right) \cdot \hat{n}=v_{0}$,

$$
p(\boldsymbol{r}) \equiv p_{n}(\boldsymbol{r})=A \int R^{-1} \exp (i k R) d S
$$

where $A=-i \omega \rho_{0} v_{0} / 2 \pi=-i p_{0} / \lambda, p_{0}=\rho_{0} c_{0} v_{0}$ is a pressure amplitude, ${ }^{20}$ and $\lambda$ is the wavelength. The subscript attached to $p$ designates the nearfield solution, but the expression is general with respect to the field at $\boldsymbol{r}$.

Farfield: At very large distances $r$,

$$
\lim _{r \rightarrow \infty} p(\boldsymbol{r}) \equiv p_{f}(\boldsymbol{r})=A \pi a^{2} \frac{2 J_{1}(k a \sin \theta)}{k a \sin \theta} \frac{\exp (i k r)}{r},
$$

where $\theta$ is the angle between the transducer axis, with unit vector $\hat{z}$ in rectangular coordinates, and direction $\hat{\boldsymbol{r}}$ to the field point, i.e., $\theta=\arccos (\hat{z} \cdot \hat{\boldsymbol{r}})$, and $J_{l}(\zeta)$ is the Bessel function of order 1 and argument $\zeta$. The directional part of the farfield amplitude is typically expressed through the beam pattern $b(\theta)=20 \log \mid 2 J_{l}(k a \sin \theta) /$ $(k a \sin \theta) \mid$.

Axial field: On the transducer axis $r=(0,0, z)$, and

$$
\begin{aligned}
p_{a x}(z)= & A \frac{4 \pi}{k} \sin \left\{\mathrm{k}\left[\sqrt{z^{2}+a^{2}}-\mathrm{z}\right] / 2\right\} \\
& \times \exp \left\{\mathrm{ik}\left[\sqrt{z^{2}+a^{2}}+\mathrm{z}\right] / 2\right\} .
\end{aligned}
$$

In the limit that $z$ becomes very large, this reduces to $A \pi a^{2}$ $\exp (i k z) / z$, which is equivalent to the value obtained from Eq. (3) for $\theta=0$. 


\section{NUMERICAL ISSUES}

\section{A. Finite-element representation of a circular piston}

Description of the nearfield at arbitrary field positions requires numerical evaluation of the integral in Eq. (2). This is done through a Riemann summation in which the circular piston is represented by small finite elements that exactly cover the active acoustic area. The circular area is subdivided into equal-area curvilinear elements, with six in the central circular area, denoted $j=1$, and $6(2 j-1)$ quadrilateral curvilinear elements in annulus $j$. For $n$ annuli, the total number of elements is $6 n^{2}$.

For a transducer of relative size $k a$ and $n=100$, the number of elements of radial dimension $\Delta a$ per wavelength is $\lambda / \Delta a=200 \pi / k a$. For $k a=10, \lambda / \Delta a=20 \pi \approx 63$. This exceeds the criterion given by Lockwood and Willette ${ }^{43}$ that this number be at least 10 .

The vector position $\boldsymbol{r}^{\prime}$ of each element is required in the finite-summation form of the integral in Eq. (2). The respective centroid of each element is used.

\section{B. Benchmarking}

Numerical code used to evaluate $p_{n}(\mathbf{r})$ in Eq. (2) is written for the general field position $\mathbf{r}$. To benchmark the code, the result of evaluating Eq. (2) along the piston axis is compared with the exact axial solution given in Eq. (4). The number of finite elements used to represent the circular area is sufficient to ensure convergence in both real and imaginary components to within $1 \%$ in amplitude in the worst evaluated case, $z=0.01 a^{2} / \lambda$. The error decreases with increasing $z$; it is within $0.001 \%$ by $z=4 a^{2} / \lambda$.

Other benchmarking is available, for example, through analytic evaluations of $p_{f}(r, \theta)$ in Eq. (3) and $p_{a x}(r, \theta)$ in Eq. (4) in Refs. 3, 8, 20, and 21, among other prominent texts. These evaluations include the positions of maxima and minima in the farfield angular patterns and along the nearfield axis.

\section{Error characterization}

Errors can be characterized in a number of ways. Consistent with Bobber's use, ${ }^{1}$ the error $\varepsilon$ is defined thus

$$
\varepsilon=20 \log \left|p_{f}(r, \theta) / p_{n}(r, \theta)\right|,
$$

where $p_{n}$ and $p_{f}$ are evaluated as described above. It is noted that while the expression for $p_{f}$ was developed in the limit of very large $r$, it is applied at all field positions with finite $r$, described as an inward extrapolation.

\section{RESULTS AND DISCUSSION}

\section{A. Nearfield-farfield transition}

The axial field of a circular piston projector with uniform normal velocity distribution is known exactly, as stated in Sec. II. It has been published often; indeed, it is a textbook illustration of basic wave-interference phenomena, with an optical analogy too. This is exemplified by the occurrence of bright - diffraction - spots in the shadow behind an illuminated disk. ${ }^{9}$ A single example of the axial field of a circular piston transducer is given here in Fig. 1(a) for the wavenumber-radius product $k a=10$.

Less well known is the off-axis field, whose range dependence is illustrated in Fig. 1(b)-1(d) for three fixed angles, $15.9,20.0$, and $21.6 \mathrm{deg}$ for the same circular piston, with $k a=10$. These angles correspond to values of the beam pattern $b(\theta)=-10,-20$, and $-30 \mathrm{~dB}$, respectively. As the magnitude of the beam pattern value increases, so does the difference in inward-extrapolated farfield and exact nearfield values. This difference is quantified through the error $\varepsilon$ in Eq. (5).

Another way of illustrating the nature of the departure of the inward-extrapolated farfield from the exact nearfield is r/a
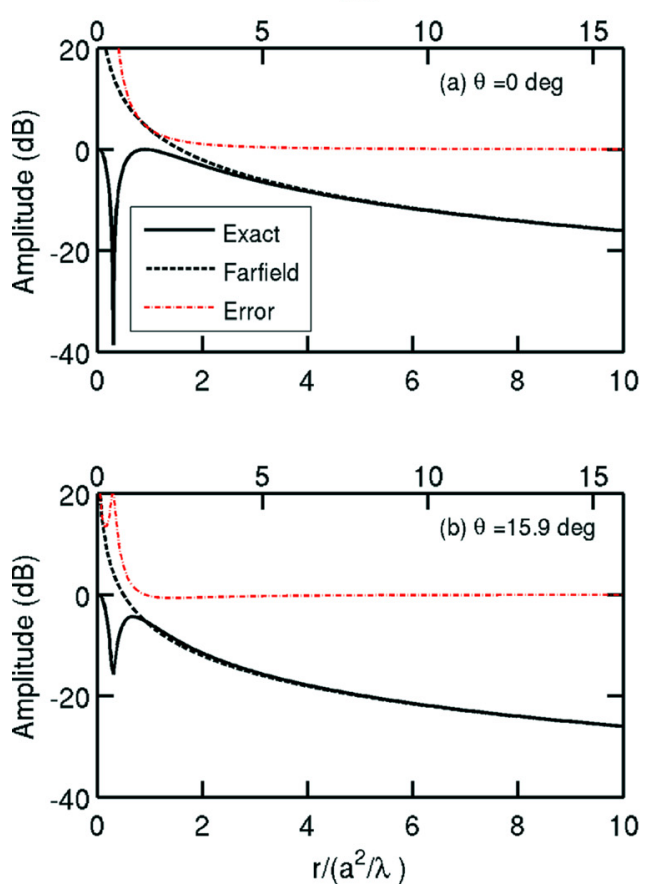

r/a
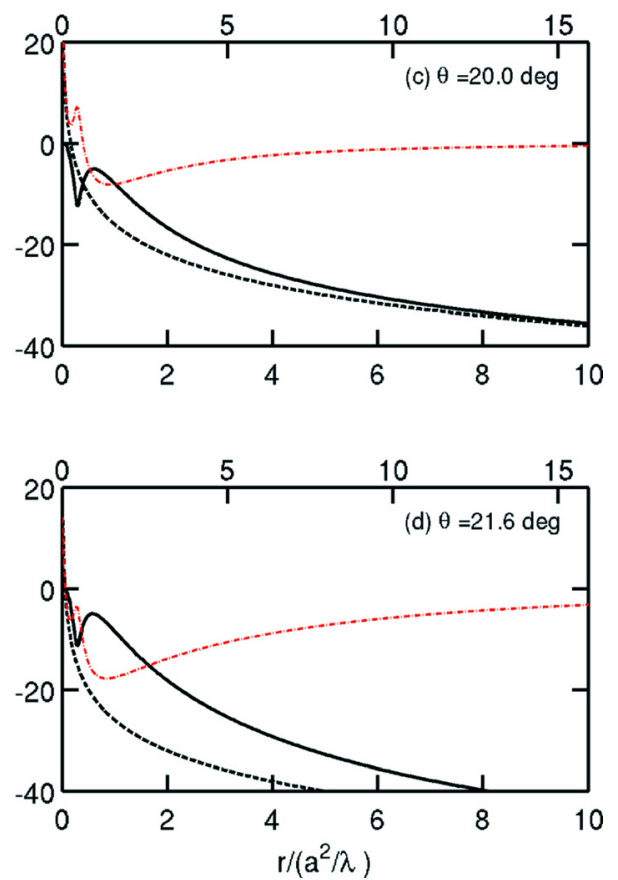

FIG. 1. (Color online) Dependence of the nearfield amplitude $\left|p_{n}(r, \theta)\right|$, inward-extrapolated farfield amplitude $\left|p_{f}(r, \theta)\right|$, and error $20 \log \left|p_{f} / p_{n}\right|$ on range $r$ for a perfectly baffled planar circular piston transducer, with uniform normal velocity distribution and wavenumber-radius product $k a=10$, where $r$ is measured from the transducer center to the field point, for each of four angles $\theta$ measured from the piston axis to the vector field position. (a) $\theta_{1}=0$, on axis. (b) $\theta_{2}=15.9 \mathrm{deg}$, for which the one-way beam pattern $b\left(\theta_{2}\right)=-10 \mathrm{~dB}$. (c) $\theta_{3}=20.0 \mathrm{deg}$, for which $b\left(\theta_{3}\right)=-20 \mathrm{~dB}$. (d) $\theta_{4}=21.6 \mathrm{deg}$, for which $b\left(\theta_{4}\right)=-30 \mathrm{~dB}$. 
shown in Fig. 2. Here, the angular dependence of the error $\varepsilon$ is shown for each of three fixed ranges: $a^{2} / \lambda, \pi a^{2} / \lambda$, and $10 a^{2} / \lambda$.

The nearfield and farfield amplitudes evidently display convergence with increasing range, with the significant exception of the troublesome null regions, referring to minima in $b(\theta)$. For a circular piston transducer, the nulls occur at the zeroes of $J_{1}(\zeta)$, namely, $\zeta \approx 3.8317$ and 7.0156. Since $\zeta=k a \sin \theta$ and $k a=10, \theta=22.5$ and $44.6 \mathrm{deg}$.

Admittedly, the scales in Figs. 1 and 2 are coarse, preventing both quantification of the degree of convergence and comparison of the kinds of error-related proximity criteria prescribed in Refs. 1 and 27. A third set of computations was performed, therefore, to increase the fineness of the discrimination in nearfield and inward-extrapolated farfield amplitudes. In Fig. 3, the range beyond which the error in inward-extrapolated farfield amplitude relative to the exact nearfield amplitude is less than 1 or $0.3 \mathrm{~dB}$ is given as a function of angle $\theta$.

In the specific case of an error bound of $0.3 \mathrm{~dB}$, in Fig. 3 (b), the inward-bounded range exceeds $20 a^{2} / \lambda=100 a / \pi$ $\approx 32 a$ for $k a=10$ over a cumulative angular span of $5.5 \mathrm{deg}$, or about $6 \%$ of the total span of $\theta$. This result rather gives the lie to the notion of simple, short-range criteria for the end of the nearfield and/or beginning of the farfield, the more so when an error bound is stated or is implicit. This analysis is repeated in Sec. IV B for specific error-related proximity criteria given in Refs. 1 and 27.
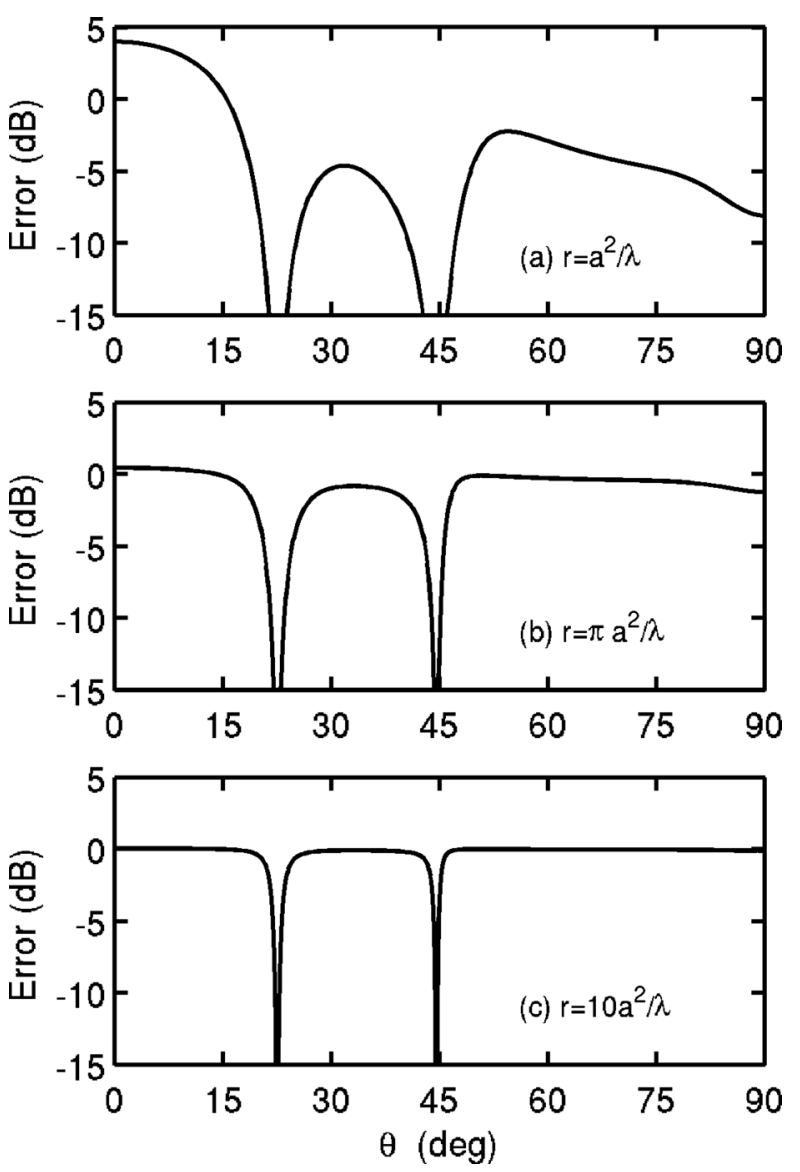

FIG. 2. Dependence of the error $20 \log \left|p_{f} / p_{n}\right|$ on angle $\theta$ for a perfectly baffled planar circular piston transducer, with uniform normal velocity distribution and wavenumber-radius product $k a=10$, at each of three fixed ranges $r$. (a) $r_{1}=a^{2} / \lambda$. (b) $r_{2}=\pi a^{2} / \lambda$. (c) $r_{3}=10 a^{2} / \lambda$.
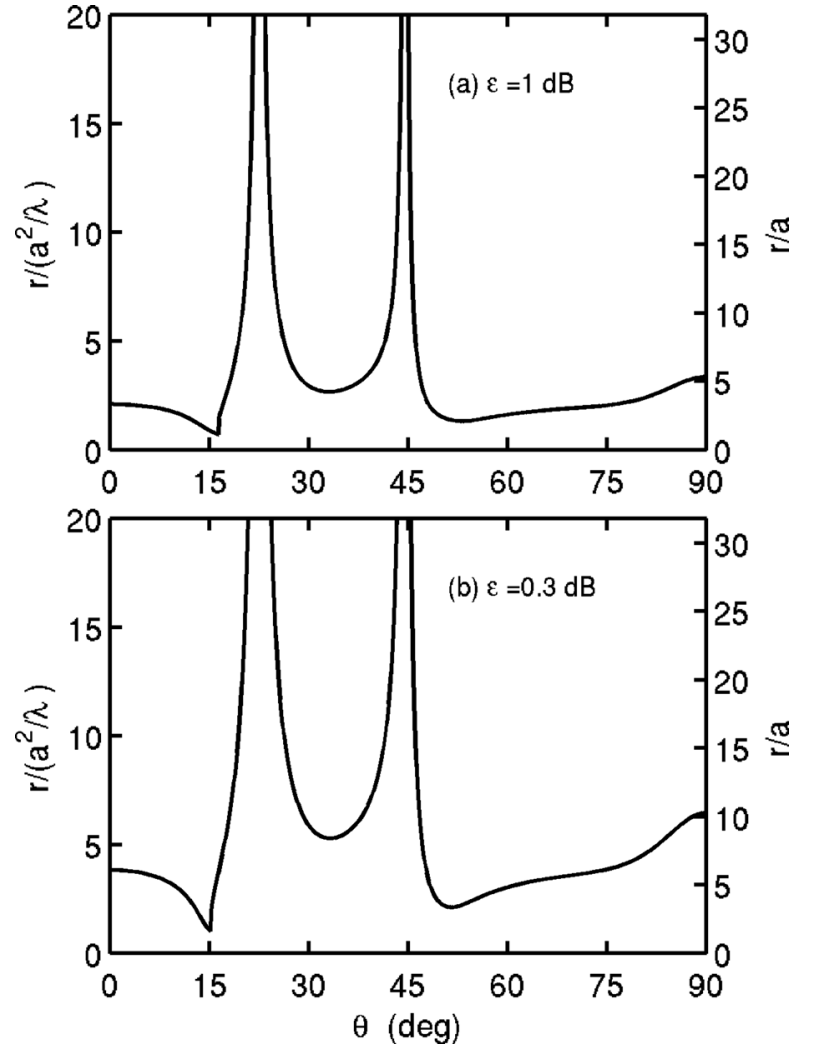

FIG. 3. Dependence of the range $r$ beyond which the error in the inwardextrapolated farfield amplitude $\left|p_{f}(r, \theta)\right|$, relative to the exact nearfield amplitude $\left|p_{n}(r, \theta)\right|$, is less than two values of the error $\varepsilon=20 \log \left|p_{f} / p_{n}\right|$. (a) $\varepsilon_{1}=1 \mathrm{~dB}$. (b) $\varepsilon_{2}=0.3 \mathrm{~dB}$.

The present analyses have been repeated for a circular piston transducer of relative size $k a=20$. The nearfield structure is more complicated, which is to be expected from the greater size relative to the wavelength. However, the results are quantitatively similar with respect to the phasebased proximity criterion and at least qualitatively similar otherwise. On axis, for example, the transition distance for a $1-\mathrm{dB}$ error is $2.1 a^{2} / \lambda=3.3 a$ for $k a=10$ and $2.0 a^{2} / \lambda=6.3 a$ for $k a=20$. Also on axis, but for a $0.3-\mathrm{dB}$ error, the transition distance is $3.8 a^{2} / \lambda=6.1 a$ for $k a=10$ and $3.6 a^{2} / \lambda$ $=11.4 a$ for $k a=20$.

It is clear from the computational results in Figs. 1-3, for $k a=10$, that there are angular regions where measurements are contraindicated, even when strictly observing errorrelated amplitude- and phase-based proximity criteria, as in Refs. 1 and 27. Given that measurements of transducer performance are still needed, and the available measurement volume may be limited, two tactics are evident: (i) derivation and application of compensation functions, or corrections, as illustrated by Bobber, ${ }^{1}$ and (ii) assignment of quality factors, or tolerances, to measurements made at ranges violating the basic error criteria. This statement is equally true of ordinary measurements made with transducers in their nearfields.

\section{B. Comparison with published error-related proximity criteria}

Several sets of error-related proximity criteria for the perfectly baffled circular piston were cited in Sec. I A. Two 
of these sets are now examined according to the Rayleighintegral model used to describe the nearfield.

For off-axis measurements, Bobber ${ }^{1}$ derived these amplitude- and phase-criteria for an error bound of $0.5 \mathrm{~dB}$ : $r \geq 10 a$ and $r \geq 4 a^{2} / \lambda$. Assuming the equality, the first of these is the more stringent. In a detailed computation of the type shown in Fig. 2 but computed for the range $r=10 a=2 \pi a^{2} / \lambda$ for $k a=10$, it was found that the $0.5-\mathrm{dB}$ error bound was exceeded over the angular ranges 18.9-27.3 and $40.4-46.3 \mathrm{deg}$, i.e., cumulatively $14.3 \mathrm{deg}$ or about $16 \%$ of the total span of $\theta$.

Also for off-axis measurements, IEC $60565^{27}$ gives these amplitude- and phase-criteria for an error bound of $0.3 \mathrm{~dB}: r>20 a$ and $r>8 a^{2} / \lambda$. Again, assuming that the equality applies, justified by the continuity of the nearfield pressure amplitude, the amplitude-criterion is the more stringent. In a computation analogous to that described above for Bobber's case, performed for $r=20 a=4 \pi a^{2} / \lambda$ for $k a=10$, it was found that the $0.3-\mathrm{dB}$ error bound was exceeded over the angular ranges $20.0-25.5$ and $42.4-45.8 \mathrm{deg}$, i.e., cumulatively $8.9 \mathrm{deg}$ or about $10 \%$ of the total span of $\theta$.

\section{Other effects}

As earlier noted, the present work addresses the problem of the nearfield-farfield transition for a planar circular piston, with uniform normal velocity distribution and with mounting in an infinite, rigid planar baffle, for measurement by an idealized point receiver. For a finite-size receiver, the problem would have to be addressed anew, as appreciated and practiced in Refs. 22-24 and 27, although for special aperture configurations in each of these cases.

Assumptions made about baffling are apparently extreme, but effectively achievable with finite physical baffles at higher frequencies. Methods exist for their quantification, beginning with Nichols. ${ }^{44}$ Keele $^{45}$ has made measurements of loudspeakers with and without baffling, noting changes in the response function due to frequencydependent effects of diffraction and directionality. These effects will also be present in the case of unbaffled acoustic piston transducers used underwater.

The assumption of a uniform normal velocity distribution on the piston surface also raises questions about applicability, given the nature of mountings of acoustically active materials, e.g., piezoelectric crystals. ${ }^{6}$ The assumption is nonetheless reasonable and useful in many cases, especially at higher frequencies. It is possible to treat cases of nonuniform normal velocity distributions through the Rayleigh integral, but in other ways too, e.g., analytically for certain distribution functions. ${ }^{2,25,26}$

Non-planar radiating surfaces have also been studied. ${ }^{45}$ In the case of loudspeakers with a conical membrane, or piston, Keele raised the question of the applicability of standard planar-piston theory but without answering this.

In general, on the subject of assumptions about baffling and normal velocity distributions, Beyer and Letcher have commented, "It is therefore a stroke of good fortune that the results of the analysis... [based on the Rayleigh integral evaluated for such an idealized baffled circular piston transducer]... have been quite accurately verified."

\section{SUMMARY AND CONCLUSIONS}

The nearfield of a planar circular piston, with uniform normal velocity distribution and mounting in an infinite planar rigid baffle, is indeed complicated, but amenable to modeling by the Rayleigh integral. The particular error analyses performed in this work, by numerical evaluation of the Rayleigh integral, have quantified departures in the inwardextrapolated farfield solution from the essentially exact nearfield solution of the harmonic wave equation. These error analyses have illustrated a method that can be applied much more generally to discriminate the nearfield-farfield transition of suitably baffled, planar acoustic transducers with known normal velocity distribution.

Current amplitude- and phase-based proximity criteria for the nearfield-farfield transition are limited in applicability. It is recommended that compensation functions, or corrections, be derived by modeling with the Rayleigh integral and applied to measurements made of the performance of acoustic transducers, as well as to measurements made with the same, in their nearfields. It is also suggested that quality factors, or tolerances, be assigned to measurements made at nearfield positions.

It is remarked that the Rayleigh-integral method does not distinguish between amplitude- and phase-based proximity criteria. Indeed, for circular piston transducers the two are linked by the equality $a=2 \pi(k a)^{-1} a^{2} / \lambda$.

The present results and conclusions also apply to the reciprocal cases of measurements made of the performance of acoustic transducers and ordinary measurements made with such devices, when acting as receivers of sound emanating from a point source. The principle of reciprocity, which is invoked, applies strictly to the same conditions of baffling and acoustic sensitivity.

\section{ACKNOWLEDGMENTS}

S. P. Robinson and P. D. Theobald are thanked for discussions on nearfield measurement criteria; A. D. Pierce, for general discussions on the nearfield. G. Hayman and R. Drake are both thanked for information on circular piston transducers being used in their respective laboratories. Advice on MATLAB matters from C. Bassett and A. Newhall is gratefully acknowledged.

${ }^{1}$ R. J. Bobber, Underwater Electroacoustic Measurements (Naval Research Laboratory, Washington, DC, 1970), pp. 121-133.

${ }^{2} \mathrm{H}$. Stenzel, Leitfaden zur Berechnung von Schallvorgängen (Guide to the Calculation of Sound Waves) (Springer, Berlin, 1939), pp. 77-79.

${ }^{3} \mathrm{~S}$. N. Rschevkin, A Course of Lectures on the Theory of Sound, transl. O. M. Blunn, edited by P. E. Doak (Pergamon, Macmillan, New York, 1963), pp. $433-447$.

${ }^{4}$ E. Skudrzyk, The Foundation of Acoustics: Basic Mathematics and Basic Acoustics (Springer, Wien, 1971), pp. 631-633.

${ }^{5}$ A. O. Williams, Jr., "The piston source at high frequencies," J. Acoust. Soc. Am. 23, 1-6 (1951).

${ }^{6}$ R. T. Beyer and S. V. Letcher, Physical Ultrasonics (Academic, New York, 1969), pp. 12-16.

${ }^{7}$ J. Zemanek, "Beam behavior within the nearfield of a vibrating piston," J. Acoust. Soc. Am. 49, 181-191 (1971). 
${ }^{8}$ A. D. Pierce, Acoustics: An Introduction to its Physical Principles and Applications (McGraw-Hill, New York, 1981), pp. 225-227, 214-215, 231-233.

${ }^{9}$ M. Born and E. Wolf, Principles of Optics: Electromagnetic Theory of Propagation, Interference and Diffraction of Light, 7th (expanded) ed. (Cambridge University Press, Cambridge, 1999), pp. 425-430, 417.

${ }^{10}$ L. L. Beranek, Acoustic Measurements (Wiley, New York, 1949), p. 120.

${ }^{11}$ D. Stansfield, Underwater Electroacoustic Transducers: A Handbook for Users and Designers (Bath University Press, Claverton Down, Bath, 1991; reprinted by Peninsula Press, Los Altos Hills, CA), pp. 171-172.

${ }^{12}$ L. E. Kinsler and A. R. Frey, Fundamentals of Acoustics (Wiley, New York, 1950), pp. 184-187.

${ }^{13}$ L. E. Kinsler, A. R. Frey, A. B. Coppens, and J. V. Sanders, Fundamentals of Acoustics, 3rd ed. (Wiley, New York, 1982), pp. 187-188.

${ }^{14}$ L. E. Kinsler, A. R. Frey, A. B. Coppens, and J. V. Sanders, Fundamentals of Acoustics, 4th ed. (Wiley, New York, 2000), pp. 179-181.

${ }^{15}$ R. J. Urick, Principles of Underwater Sound for Engineers (McGraw-Hill, New York, 1967), p. 37.

${ }^{16}$ R. J. Urick, Principles of Underwater Sound, 2nd ed. (McGraw-Hill, New York, 1975), p. 46.

${ }^{17}$ R. J. Urick, Principles of Underwater Sound, 3rd ed. (McGraw-Hill, New York, 1983), pp. 71-73.

${ }^{18}$ C. S. Clay and H. Medwin, Acoustical Oceanography: Principles and Applications (Wiley, New York, 1977), pp. 153-155.

${ }^{19}$ H. Medwin and C. S. Clay, Fundamentals of Acoustical Oceanography (Academic, Boston, 1998), pp. 142-143.

${ }^{20}$ D. T. Blackstock, Fundamentals of Physical Acoustics (Wiley, New York, 2000), pp. 446-457.

${ }^{21}$ C. H. Sherman and J. L. Butler, Transducers and Arrays for Underwater Sound (Springer, New York, 2007), pp. 530-531, 448-449, 455-456.

${ }^{22}$ G. A. Sabin, "Calibration of piston transducers at marginal test distances," J. Acoust. Soc. Am. 36, 168-173 (1964).

${ }^{23}$ R. J. Bobber and G. A. Sabin, "Cylindrical wave reciprocity parameter," J. Acoust. Soc. Am. 33, 446-451 (1961).

${ }^{24}$ V. I. Sorokin, "Determination of the sensitivity of cylindrical transduces in marginal-distance calibrations," Sov. Phys. Acoust. 19, 274-275 (1973).

${ }^{25}$ M. Greenspan, "Piston radiator: Some extensions of the theory," J. Acoust. Soc. Am. 65, 608-621 (1979).

${ }^{26}$ R. M. Aarts and A. J. E. M. Janssen, "Sound radiation quantities arising from a resilient circular radiator," J. Acoust. Soc. Am. 126, 1776-1787 (2009).

${ }^{27}$ IEC60565, "Underwater acoustics-Hydrophones-Calibration in the frequency range $0.01 \mathrm{~Hz}$ to $1 \mathrm{MHz}$," IEC 60565 ed2.0 (International Electrotechnical Commission, Geneva, 2006).
${ }^{28}$ ANSI/ASA S1.20-2012, “American National Standard: Procedures for Calibration of Underwater Electroacoustic Transducers" (Acoustical Society of America, Melville, NY, 2012).

${ }^{29}$ L. J. Ziomek, Fundamentals of Acoustic Field Theory and Space-Time Signal Processing (CRC Press, Boca Raton, FL, 1995), pp. 405-415.

${ }^{30}$ W. L. Nyborg and R. B. Steele, "Nearfield of a piston source of ultrasound in an absorbing medium," J. Acoust. Soc. Am. 78, 1882-1891 (1985).

${ }^{31}$ C. H. Sherman, "Effect of the nearfield on the cavitation limit of transducers," J. Acoust. Soc. Am. 35, 1409-1412 (1963).

${ }^{32}$ K. G. Foote, “Acoustic sampling volume," J. Acoust. Soc. Am. 90, 959-964 (1991).

${ }^{33}$ O. R. Godø, K. G. Foote, J. Dybedal, E. Tenningen, and R. Patel, "Detecting Atlantic herring by parametric sonar," J. Acoust. Soc. Am. 127, EL153-EL159 (2010).

${ }^{34}$ P. J. Westervelt, "Parametric acoustic array," J. Acoust. Soc. Am. 35, 535-537 (1963).

${ }^{35}$ K. G. Foote, D. T. I. Francis, and P. R. Atkins, "Calibration sphere for low-frequency parametric sonars," J. Acoust. Soc. Am. 121, 1482-1490 (2007).

${ }^{36} \mathrm{~K}$. G. Foote, "Range compensation for backscattering measurements in the difference-frequency nearfield of a parametric sonar," J. Acoust. Soc. Am. 131, 3698-3709 (2012).

${ }^{37}$ R. H. Mellen and M. B. Moffett, "A numerical method for calculating the nearfield of a parametric acoustic source," J. Acoust. Soc. Am. 63, 1622-1624 (1978)

${ }^{38}$ M. B. Moffett and R. H. Mellen, "Nearfield characteristics of parametric acoustic sources,” J. Acoust. Soc. Am. 69, 404-409 (1981).

${ }^{39}$ A. L. Van Buren, M. D. Jevnager, and A. C. Tims, "A 5- to 50-kHz synthetic cylindrical nearfield calibration array," J. Acoust. Soc. Am. 77, 1927-1932 (1985).

${ }^{40}$ A. L. Van Buren, "Spherical nearfield calibration array for threedimensional scanning," J. Acoust. Soc. Am. 85, 2655-2660 (1989).

${ }^{41}$ A. L. Van Buren, "Near-field transmitting and receiving properties of planar near-field calibration arrays," J. Acoust. Soc. Am. 89, 1423-1427 (1991).

${ }^{42}$ J. W. Strutt, The Theory of Sound, 2nd ed. revised and enlarged (Dover, New York, 1945), Vol. II, pp. 106-109.

${ }^{43}$ J. C. Lockwood and J. G. Willette, "High-speed method for computing the exact solution for the pressure variations in the nearfield of a baffled piston,” J. Acoust. Soc. Am. 53, 735-741 (1973); 54, 1762(E) (1973).

${ }^{44}$ R. H. Nichols, Jr., "Effects of finite baffles on response of source with back enclosed," J. Acoust. Soc. Am. 18, 151-154 (1946).

${ }^{45}$ D. B. Keele, Jr., "Low-frequency loudspeaker assessment by nearfield sound-pressure measurement," J. Audio Eng. Soc. 22, 154-162 (1974). 